\title{
O DISSENSO ENTRE ATORES PÚBLICOS E PRIVADOS ENVOLVIDOS EM PARQUES TECNOLÓGICOS EM OPERAÇÃO NO BRASIL
}

\section{The dissent between public and private actors involved in Science Parks in operation in Brazil}

\section{Lindsay Teixeira Sant'Anna}

Universidade Federal de Lavras. e-mail: lindsaysantanna@gmail.com

\section{Dany Flávio Tonelli}

Universidade Federal de Lavras.e-mail:danytonelli@gmail.com

\section{Teresa Cristina Monteiro Martins}

Universidade Federal de Lavras. e-mail:teresacristina.ufla@gmail.com

Luiz Marcelo Antonialli

Universidade Federal de Lavras.e-mail:Imantonialli@dae.ufla.br

\section{RESUMO}

Diante do crescimento das iniciativas de parques tecnológicos no país e da multiplicidade de atores envolvidos, o presente estudo tem por objetivo investigar as diferenças de percepções e de práticas entre atores públicos e os privados envolvidos nesses empreendimentos. Por meio da análise de clusters e da análise discriminante, com utilização do programa Statistical Package for the Social Sciences (SPSS), constatou-se significativas diferenças de percepções quanto à importância dada por atores públicos e privados às variáveis da gestão do conhecimento, confiança e investimento. Quanto às ações, os atores públicos afirmam realizar práticas de confiança, interdependência e ampla participação nos processos decisórios com uma multiplicidade de envolvidos, o que não é confirmado pelos atores privados. Assim, apesar do crescimento do número de parques tecnológicos em operação no país revelar uma predisposição à colaboração, o dissenso constatado entre as percepções e as práticas colaborativas realizadas por atores públicos e privados podem comprometer a viabilidade desses empreendimentos no futuro.

Palavras-Chave: colaboração, atores públicos, atores privados, análise discriminante, análise de clusters.

\section{ABSTRACT}

In view of the growth of Science Parks initiatives in the country and the multiplicity of actors involved, this study aims to investigate the differences in perceptions and practices between public and private actors involved in these ventures. Through the analysis of clusters and the discriminant analysis, using Statistical Package for the Social Sciences (SPSS) program, significant differences in perceptions were observed regarding the importance given by public and private actors to the variables: knowledge management, trust and investment. As for the actions, the public actors affirm to practice practices of trust, interdependence and wide participation in the decision processes with a multiplicity of involved, which is not confirmed by the private actors. Thus, despite the growth in the number of Science Parks in operation in the country revealing a predisposition to collaboration, the disagreement between the perceptions and collaborative practices carried out by public and private actors can compromise a challenge for the sustainability and durability of these enterprises.

Key Words: collaboration, public actors, private actors, discriminant analysis, cluster analysis. 


\section{INTRODUÇÃO}

Com a proposta de promover o desenvolvimento tecnológico local por meio de processos inovativos, os parques tecnológicos são fruto das articulações entre diversos atores públicos e privados, como os poderes locais, empresas, universidades e centros de pesquisa (LACERDA; FERNANDES, 2015; PESSOA et al., 2012; TONELLI; MARQUESINI; ZAMBALDE, 2015; VEDOVELLO, JUDICE, MACULAN, 2006).

Apesar do recente movimento e experiências brasileiras no campo de criação desses empreendimentos, (ZOUAIN; PLONSKI, 2006) alguns benefícios são destacados na literatura como: a boa infraestrutura oferecida pelos parques, a estimulação à inovação empresarial e à gestão do conhecimento decorrentes da parceria entre empresas e academia (HANSEN et al, 2012; HOBBS; LINK; SCOTT, 2017; LAIMER, 2015; LACERDA; FERNANDES, 2015), o desenvolvimento de vínculos entre diferentes organizações (HERVÁS-OLIVER; ALBORS-GARRIGOS, 2009), de forma a identificar interesses comuns que podem levar a projetos conjuntos (GUILLAIN; HURIOT, 2001).

No entanto, Laimer (2015) e Schmidt e Balestrin (2014) apontam que ainda não está claro quais elementos fazem parte das relações interorganizacionais que envolvem universidades, empresas e governo, no contexto de parques científicos e tecnológicos. Assim, os autores assinalam a necessidade de mais pesquisas neste campo. Somando-se a isso, há poucos estudos brasileiros que buscam compreender e/ou analisar as relações entre os envolvidos em parques tecnológicos sob a ótica da governança colaborativa (GC), que é o foco deste estudo. Destaca-se os recentes estudos de Abbud e Tonelli (2018) e Tonelli, Costa e Sant'Anna (2018), cujas análises se concentraram na compreensão dos constructos teóricos da GC em parques tecnológicos no Estado de Minas Gerais. Já o presente estudo é fruto de uma pesquisa empírica realizada com 32 parques em operação em todas as regiões do país, com o uso da lente teórica da governança colaborativa.

A GC é um tipo distinto dos outros tipos de governança já explorados na literatura brasileira, como a corporativa e a governança em rede. A principal diferença que a governança colaborativa apresenta frente a esses outros regimes, é que ela tem o condão de buscar o consenso entre os envolvidos em uma política pública, por meio de um processo deliberativo inclusivo, onde haja decisões permeadas e influenciadas por debates entre os interessados (ANSELL; GASH, 2007; FOSTER-FISHMAN et al., 2001; NEWMAN et al. 2004; FREEMAN,1997; WEBER; KHADEMIAN, 2008).

Se a GC é, na compreensão de Ansell e Gash (2008), um processo caracterizado por etapas não lineares de interação entre os atores, na busca por objetivos em comum, compartilhados entre público e privado, as interações que ocorrem nos parques tecnológicos seguem este mesmo raciocínio. Contudo, para que a colaboração ocorra é imprescindível que os envolvidos se sintam cocriadores responsáveis pelos processos colaborativos compostos por determinadas características imprescindíveis para a durabilidade da colaboração (ANSELL; GASH 2008; EMERSON; NABATCHI, 2015; SANT'ANNA; TONELLI; ABBUD, 2016; TONELLI; SANT'ANNA; ABBUD, 2018).

Destrinchando em miúdos, o processo brasileiro de implantação de parques tecnológicos funciona assim: o poder público atua como um facilitador para as parcerias público-privadas, por meio de investimentos financeiros e fiscais tanto aos entes públicos quanto aos privados e aos empresários. Estes últimos, necessitados de tecnologia para a inovação de seus produtos e serviços, direcionam essa demanda às universidades e aos institutos de pesquisas que, por sua vez, realizam as pesquisas que contribuem para o avanço da sociedade em todos os setores, dando origem ao compartilhamento do conhecimento entre setores público e privado (STEINER; CASSIN; ROBAZZI, 2008; ZEN, 2005). Para que tudo isso aconteça há a necessidade de participação, colaboração e diálogo entre todos os envolvidos na busca por soluções, reduzindo o poder centralizador do governo e aumentando a transparência das ações (MOREIRA et al., 2014). As dificuldades de estabelecer interações bem sucedidas entre todos os atores envolvidos e de promover consensos mínimos sobre série de controvérsias que os envolvem podem se tornar um risco ao sucesso desses empreendimentos coletivos no futuro.

Sendo assim, diante da multiplicidade de atores envolvidos e da presença de grupos de atores públicos e privados, quais são as diferenças de percepções e de práticas entre os atores públicos e os privados no que tange ao processo colaborativo nos parques 
tecnológicos instalados no Brasil? O objetivo geral desse estudo, então, é investigar tais diferenças e como elas se manifestam em relação às variáveis da GC identificadas na literatura.

Para o alcance dos objetivos do estudo, após a exploração sucinta da temática dos parques tecnológicos no Brasil sob a ótica da GC relata-se, na sequência, os procedimentos metodológicos do presente estudo para dar início às demonstrações dos resultados e discussões. Ao fim, apresentam-se as considerações finais da pesquisa.

\section{PARQUES TECNOLÓGICOS E GOVERNANÇA COLABORATIVA}

Os parques tecnológicos constituem-se de redes de atores públicos e privados capazes de construir relações de troca e parceria fomentando os sistemas de inovação (SILVA; SUASSUNA; MACIEL, 2009; PESSOA et al, 2012)..Em um estudo apresentado pela ANPROTEC (2014) apontou-se que, em 2013, o Brasil possuía um total de 94 iniciativas de parques tecnológicos espalhados no Brasil, sendo que destes, 28 já estavam em operação (ANPROTEC, 2017a). Um outro estudo da Anprotec, de 2015, aponta para uma expectativa de que o Brasil terá um total de 95 parques tecnológicos em operação até o ano de 2030 (ANPROTEC, 2017b).

Esse crescimento leva a crer que tanto o setor privado quanto o público vislumbram benefícios na criação dos parques tecnológicos no Brasil, como a boa infraestrutura oferecida pelos parques; a estimulação à inovação empresarial decorrente da parceria entre empresas e academia (HANSEN et al., 2012; LAIMER, 2015; LACERDA; FERNANDES, 2015), o desenvolvimento de vínculos entre diferentes organizações (Hervás-Oliver e Albors-Garrigos, 2009; VÁSQUEZ-URRIAGO, BARGE-GIL, RICO, 2016) relacionamentos sustentáveis entre as partes interessadas, redução nos custos de transação, vantagem competitiva, boa reputação e agregação de valor no produto ou serviço (CHILD, FAULKNER; TALLMAN, 2005; DYER; SINGH, 1998; TENCATI; ZSOLNAI, 2009).

Se o caminho que se apresenta para a ação pública é pela permeabilidade das fronteiras e pela aproxi- mação entre atores governamentais, da sociedade civil e da economia, algo fundamental para a consolidação do cenário ideal está em prestar atenção na qualidade das relações estabelecidas entre Estado, sociedade e mercado. Tanto é assim, que o sentido dado aos mecanismos de governança do setor público se alterou nos últimos anos. Inicialmente, a governança estava relacionada mais com os aspectos operacionais das máquinas administrativas (RHODES, 1996; PETERS, 1997). Atualmente, a governança traduz também a preocupação com a articulação entre os atores e a constituição de arranjos institucionais de políticas públicas (DENHARDT, 2012).

Tencati e Zsolnai (2009) ressaltam que as estratégias colaborativas estão diretamente ligadas às práticas de responsabilidade corporativa e as políticas públicas devem ser estabelecidas exatamente nesse contexto de estratégias competitivas mais amplas que a colaboração oferece. Os autores entendem que há um cenário de competitividade responsável quando empresas estão em um regime de colaboração.

Dentro desse contexto de arranjos institucionais e de colaboração, percebe-se que os parques tecnológicos brasileiros merecem maiores investigações, principalmente, no que se refere aos elementos de colaboração percebidos pelos envolvidos. Isso porque defende-se que a governança colaborativa é a estratégia que mais se aproxima como possibilidade de análise para a implementação de parques tecnológicos. A política pública de criação de parques tecnológicos é uma estratégia colaborativa, que tem sido adotada no Brasil, para a promoção do desenvolvimento local e promoção da inovação por meio de parcerias público -privadas. Tal estratégia só é possível em uma proposta de governança onde o Estado não se sobreponha sobre os demais, mas ocupe o lugar de um ator-parceiro estabelecendo uma relação de colaboração com universidades e empresas.

Para a implantação de estratégias colaborativas em busca de inovação, alguns elementos são indispensáveis para o processo de colaboração na iniciativa privada como a multiplicidade de parceiros, a construção da confiança, o diálogo e a participação entre os envolvidos, a busca pelo consenso, a partilha dos investimentos, $\mathrm{o}$ comprometimento e a transparência (APPLEYARD; CHESBROUGH, 2017; CHILD, FAULKNER; TALLMAN, 2005; TENCATI; ZSOLNAI, 2009). 
No setor público, o regime colaborativo propõe uma união de esforços públicos e privados na solução de problemas públicos e/ou para a criação de novas políticas públicas, prezando por componentes relacionais tais como: engajamento com os princípios, motivação compartilhada, capacidade de ação conjunta, aprendizagem social mútua e interação entre grupos de interesse (ANSELL; GASH 2008; EMERSON;NABATCHI, 2015; EMERSON; NABATCH; BALOGH, 2012; KALLIS; KIPARSKY; NORGAARD, 2009; MAH; HILLS, 2014; PURDY, 2012).. Choi e Robertson (2014) sugerem conceituar GC como sendo uma tomada de decisão baseada em um consenso deliberativo, que envolva as partes interessadas de vários setores com seus diferentes interesses e poderes, organizado de uma forma que permita resolver problemas públicos complexos, que não poderiam ser tratados somente pelo governo. Portanto, segundo Robertson e Choi (2012), o mérito da governança colaborativa está exatamente nesta oportunidade que as partes possuem de influenciar o processo de decisão, o que não acontece em um modelo tradicional burocrático que se restringe a um processo meramente consultivo. Sendo assim, para a criação, implantação e operação de parques tecnológicos é necessária a atuação conjunta entre múltiplos atores públicos e privados, cada qual com seu papel e todos com o foco na criação de ambientes inovadores que possam proporcionar ganhos múltiplos aos envolvidos e à região onde os parques estão instalados.Nesse contexto, a governança colaborativa se encaixa perfeitamente como regime adotável em parques tecnológicos onde a colaboração é condição para a sustentabilidade dos empreendimentos. Empresas que optam pela adoção de estratégias colaborativas "compartilham estruturas de propriedade mais democráticas, sistemas de governança mais amplos e equilibrados e uma visão mais abrangente das metas e desempenhos organizacionais" (TENCATI; ZSOLNAI, 2009; p.367).

Apesar das poucas pesquisas sobre governança colaborativa em parques tecnológicos, autores como Saavedra e Budd (2009); Mah e Hills (2014); Scott (2015) buscaram em seus estudos o entendimento da colaboração em um processo de aprendizagem que incluía a incorporação de universidades e instituições de pesquisa, para se alcançar soluções de longo prazo para questões ambientais, como alterações climáticas e inovação tecnológica no mercado de energia. Percebe-se, então, que a governança colaborativa possui grande espaço para discussão em arranjos entre atores públicos e privados na busca pela inovação através de novas formas de governança. Isso não quer dizer que não haja desafios a serem enfrentados no processo de colaboração em parques tecnológicos. Questão como a interdependência, a construção da confiança e a gestão do conhecimento são apontados na literatura como questões tradicionalmente controversas na cooperação tecnológica entre atores públicos e privados.

\subsection{Interdependência, confiança e gestão do conhecimento: desafios para a colaboração tecnológica}

Nos parques tecnológicos, a colaboração tecnológica é viabilizada pela união entre governo, universidade e empresas de tal forma que as inovações não se efetivariam apenas com a iniciativa do Estado (ABDALLA; CALVOSA; BATISTA, 2009). Ou seja, a interdependência é essencial para que os envolvidos percebam a necessidade de estarem em um processo colaborativo. A ideia é convergir as três dinâmicas existentes nesse processo: transformações institucionais (que ocorrem na esfera de governo), mecanismos evolutivos (advindos das forças de mercado) e a nova posição da universidade. Tudo isso deve acontecer de tal forma que não se observe somente a relação entre universidade, indústria e governo, mas também as transformações internas ocorridas, em cada uma dessas esferas, quando em regime de cooperação (ETZKOWITZ; LEYDESDORFF, 2000). Assim, as parcerias entre universidades e empresas acabam por disseminar conhecimentos científicos e técnicos, culminando em novos processos de inovação e em um mercado competitivo local (ETZKOWITZ; LEYDESDORFF, 2000; RODRIGUES; MELO, 2013). Para se chegar a esse estágio, o grau de confiança deve estar alto e consolidado de tal forma que possa haver o compartilhamento do conhecimento dos vários atores na construção de outros conhecimentos e aprimoramento da atuação da rede. Para Weber, Lovrich, e Gaffney (2007) na medida em que existem elevados níveis de confiança entre as partes, as parcerias colaborativas provavelmente serão mais 
sustentáveis ao longo do tempo e mais eficazes na resolução de problemas.

No entanto, essas formas de parceria entre universidades e setor privado, ainda, são escassas em países da América Latina, como o Brasil (ALMEIDA, 2002). No Brasil, Abdalla, Calvosa e Batista (2009) ressaltam que a transferência do conhecimento gerado em pesquisas nas universidades, ainda, não tem sido revertido em ações concretas à sociedade. O resultado disso são pesquisas acadêmicas não aplicadas à realidade e ao interesse social e a descontinuidade de parcerias entre atores públicos e privados.

Existem duas formas de se realizar a transferência de tecnologia entre universidade e setor privado: por meio de contratos de licenciamento e transferências de tecnologia e pela criação, pela academia, de incubadoras de empresas (OLIVEIRA, 2006). Um desdobramento dessas parcerias, segundo Oliveira (2006, p. 207), é a figura dos parques tecnológicos que promovem a interação entre universidade e setor privado "na realização de atividades passíveis de gerar novos produtos e serviços baseados no conhecimento". Apesar dos aspectos positivos dessa transferência de tecnologia, também chamada de capitalização da tecnologia, Almeida (2002) alerta que, para a absorção e difusão do conhecimento, a empresa precisa ter pessoal capacitado e uma organização apropriada para se criar novas formas de produção que incluam os saberes técnicos compartilhados pela universidade, a fim de se evitar uma pseudotransferência de tecnologia. Muitas vezes, sob o ponto de vista acadêmico, a parceria promoveu o desenvolvimento e a transferência de tecnologia, mas, na prática, a falta de planejamento e observação das peculiaridades locais, faz com que as empresas não deem continuidade ao processo de inovação (RODRIGUES; MELO, 2013).

Moeliodihardjo, Soemardi, Brodjonegoro e Hatakenaka (2012) alertam ainda para algumas barreiras a serem trabalhadas nessas parceria entre universidade e empresas, como: o isolamento nas estratégias de pesquisa de algumas universidades em relação à indústria; o preconceito de alguns acadêmicos diante da visão eminentemente econômica das empresas; a extrema burocracia existente em algumas instituições tornando os processos de parceria mais lentos e a falta de entendimento, por parte da academia, das dificuldades enfrentadas pela indústria.
Há a necessidade de se criar um ambiente de envolvimento e comprometimento entre academia e empresa, que equilibre os benefícios para ambas as partes, sem exploração ou menosprezo por parte de nenhum parceiro. Na visão de Almeida (2002), há a necessidade, portanto, de se criar uma conexão entre universidade e setor produtivo, para que os projetos logrem êxito.

Apesar de todas as dificuldades, tanto o setor privado quanto o público vislumbram benefícios com a criação dos parques tecnológicos no Brasil. Alguns benefícios são destacados na literatura como a estimulação à inovação empresarial, decorrente da parceria entre empresas e academia, a boa infraestrutura (segurança, serviços e estacionamentos) oferecida pelos parques e até mesmo a valorização dos bens imobiliários localizados em bairros sedes dos empreendimentos (HANSEN; BECKER; NEFF; MELLO, 2012; LAIMER, 2015; LACERDA; FERNANDES, 2015). Ademais, o desenvolvimento de relacionamentos mais longos nos parques incentiva o compartilhamento e a partilha de conhecimentos mais valiosos entre os integrantes do grupo, resultando em um melhor ajuste entre expectativas e resultados, maior confiança e retornos crescentes da colaboração (IZUSHI, 2003; ABRAMOVSKY; SIMPSON, 2011).

\section{METODOLOGIA}

O presente estudo, quanto a sua natureza é de caráter descritivo caracterizando-se como uma pesquisa quantitativa. O método de coleta de dados escolhido para este estudo foi a aplicação de um questionário de perguntas estruturadas, por meio de ferramenta online. O questionário apresentou uma primeira parte, em que o entrevistado, considerando o parque tecnológico do qual fazia parte, deveria informar, na primeira parte, sua percepção sobre o grau de importância de cada uma das 15 (quinze) categorias de GC identificadas na literatura (SANT'ANNA; TONELLI; ABBUD, 2016; TONELLI; SANT'ANNA; ABBUD, 2018) (veja Tabela 1). A síntese dessas categorias é decorrente de amplo estudo sistemático da literatura acerca da Governança Colaborativa realizados pelos autores mencionados. 
Tabela 1 Categorias utilizadas na construção do questionário

\begin{tabular}{|c|c|c|}
\hline Estágios & Categorias & Influências sobre a qualidade de: \\
\hline \multirow{6}{*}{ 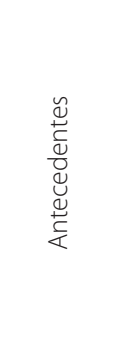 } & Múltiplos atores & Processo decisório, acesso a recursos. \\
\hline & Objetivos comuns & Ganhos coletivos, equilíbrio de poder. \\
\hline & Liderança & Alcance de resultados, resolução de conflitos, inclusão de atores e motivação. \\
\hline & Regras preliminares & Comportamento e papel dos atores, relacionamento entre atores. \\
\hline & Interdependência & Habilidades, acesso a informação. \\
\hline & Investimentos iniciais & Recursos tangíveis e intangíveis. \\
\hline \multirow{6}{*}{ 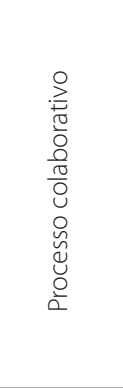 } & Processo deliberativo inclusivo & Processo democrático, participação ativa, legitimidade do processo decisório. \\
\hline & Comprometimento com o processo & $\begin{array}{l}\text { Manutenção da colaboração e percepção de coalisão, motivação permanente, } \\
\text { inovação. }\end{array}$ \\
\hline & Construção da confiança & Colaboração ativa, experiências de colaboração, motivação dos atores. \\
\hline & Relacionamentos internos e externos & Co-criação, capital social, potencial e aumento da colaboração, criação de redes. \\
\hline & Construção de consenso & Comunicação, equidade de interesses, entendimentos compartilhados. \\
\hline & Gestão do conhecimento & Compartilhamento de informações, desempenho das redes. \\
\hline \multirow{4}{*}{$\begin{array}{l}\tilde{O} \\
\frac{0}{0} \\
\frac{\tilde{D}}{5} \\
\tilde{\mathscr{U}} \\
\simeq\end{array}$} & Múltiplas fontes de investimento & Novos programas, autonomia da rede, reputação. \\
\hline & Accountability & Transparência, responsabilidade dos atores, acesso à informação. \\
\hline & Discurso e prática & Relação entre discurso e prática. \\
\hline & Desenvolvimento de novas competências & Resolução de novas demandas e desafios. \\
\hline
\end{tabular}

Fonte Adaptado de Tonelli, Sant'Anna, Abbud and Souza (2018).

Na elaboração do questionário, as categorias expostas na Tabela 1 foram apresentadas para a apreciação dos sujeitos da pesquisa. Inicialmente, o grau de importância atribuído pelo respondente a cada categoria poderia ser indicado em uma escala de quatro pontos assumida como intervalar, pelas opções: "sem importância", "pouco importante", "importante" e "muito importante". Já na segunda parte, o entrevistado deveria assinalar se tais categorias eram percebidas na prática no parque tecnológico do qual ele fazia parte, utilizando uma escala de três pontos em que assinalava se tal prática acontecia, com a assertiva "sim" ou "sim, mas parcialmente" ou "não". Após a realização do pré-teste, o questionário foi enviado aos atores envolvidos nos 32 (trinta e dois) parques tecnológicos em operação no Brasil até dezembro de 2015. Os e-mails foram obtidos por meio de uma pesquisa nos sítios eletrônicos dos parques, em redes sociais e em outras ferramentas de buscas que possibilitaram a identificação dos contatos individuais de membros da gestão do parque, do poder público e da iniciativa privada ligados às iniciativas. A partir do dia 01/12/15 foram enviados 1.009 e-mails para todos os contatos dos parques identificados nas buscas via internet. Em torno de $20 \%$ (vinte por cento) dos e-mails retornaram, por motivo de seus destinatários não serem identificados (e-mails inexistentes ou cancelados). Aconteceram envios e reenvios, portanto, nos dias: 01 e 02 de dezembro de 2015; 09 e 10 de dezembro de 2015; 16 e 17 de dezembro de 2015 e dia 11 de janeiro de 2016. Conforme os respondentes fossem identificados, não eram efetuados reenvios a estes e-mails. As respostas se iniciaram em 26/11/2015 (primeira resposta) e terminaram em 21/01/2016 (última resposta).

Foram contabilizadas um total de 193 respostas, cujos respondentes foram gestores de parques tecnológicos, representantes de prefeituras, governos dos Estados, empresas instaladas, empresas parceiras, universidades, associação parceira, gestores de incubadoras, institutos federais de ensino, instituições de ensino privadas, instituto de pesquisa, instituto 
de tecnologia, fundação pública e organização não governamental (ONG). Em todos os 32 parques em operação, atores públicos e privados responderam ao questionário.

Para fins didáticos, considerou-se como atores públicos respondentes: gestores de parques tecnológicos, representantes de prefeituras, governos dos Estados, universidades, institutos federais de ensino, instituto de pesquisa, instituto de tecnologia, fundação pública e organização não governamental (ONG). Assim, os atores privados respondentes foram: empresas instaladas, empresas parceiras, associação parceira e instituições de ensino privadas.

A tabela 2 identifica os parques cujos respondentes da pesquisa pertencem e suas respectivas localizações.

A análise de cluster teve como objetivo agrupar instituições cujos membros participantes tivessem opiniões semelhantes acerca do grau de importância e das características da governança colaborativa em suas instituições.

A análise de cluster é uma técnica de estatística multivariada utilizada para classificar uma amostra de entidades, agrupando-as de acordo com as similaridades entre as entidades (HAIR JR. et al., 2005). A análise de cluster foi realizada por meio do software SPSS, utilizando-se o método hierárquico, caracterizado por possuir um desenvolvimento com uma estrutura em árvore e compreender métodos de ligação (linkage), de variância e de centroides (PEREIRA, 2004).

Conforme recomendado por Hair, et al. (2005), foi utilizado o algoritmo hierárquico e aglomerativo Ward para a realização da hierarquização, por ser o mais popularmente utilizado devido a sua dinamicidade e simplicidade. Considerando a ideia central da análise de cluster que é a divisão da população em sub-populações com características homogêneas, com variância mínima, dentro dos clusters e características heterogêneas, com variância máxima, entre os clusters e que o método Ward combina os indivíduos dentro dos cluster por meio do cálculo da distância entre os indivíduos no cluster, foi calculado também o Quadrado da Distância Euclidiana, recomendada para os métodos de agrupamento centroide e Ward (HAIR JR. et al., 2005). Detalhando-se o método, o Quadrado da Distância Euclidiana entre os casos (i e j) é o somatório dos quadrados das diferenças entre os valores i e j para cada uma das variáveis, existindo, para tanto, $\mathrm{p}$ variáveis.

Realizado o agrupamento dos respondentes, foi necessária a identificação de quais fatores discriminavam esses grupos, estabelecendo, assim, a diferença entre eles. Para tecer conclusões acerca dessas diferenças foi realizada a análise discriminante, tmbém por meio do software SPSS.

\section{ANÁLISE DOS RESULTADOS}

Por meio da análise de cluster pelo método hierárquico Ward's, foi possível identificar dois grupos (clusters), sendo o Grupo 1 composto por 87 respondentes $(44,8 \%)$ e o Grupo 2 por 106 (54,6\%). Isso permitiu reunir em dois grupos quase a totalidade das respostas (193 de 194).

A análise confirmou a hipótese geral de que há divergências pontuais entre o posicionamento adotado pelos respondentes representantes do poder público e os respondentes do setor privado. A tabela de classificação de resultados do SPSS informou ainda que $93,8 \%$ dos casos foram corretamente classificados considerando a existência dos dois grupos distintos, podendo-se considerar válida a classificação.

A primeira constatação realizada a partir da análise do perfil das empresas classificadas nos grupos 1 e 2 é que a maioria dos respondentes vinculados à instituições públicas estão classificados no grupo 1, enquanto a maioria vinculada à instituições privadas está classificada no grupo 2.

A tabulação cruzada utilizando-se como parâmetros os valores resultantes do método Ward e cada uma das variáveis de importância ou de ações realizadas pela cooperativa, resultou na definição do perfil dos grupos. O grupo 1 pode ser identificado por atribuir menor importância às características da governança colaborativa do que o grupo 2. Entretanto, quando se analisa as ações desenvolvidas cujos respondentes dos grupos afirmam desenvolver, o grupo 2, embora enfatize mais a importância das ações em GC, tende a realizar menos ações que levam à GC. Enquanto os membros do grupo 1 afirmam realizar mais atividades consideradas geradoras da GC. Essa comparação do perfil dos grupos está descrita na Tabela 3 . 
Lindsay Teixeira Sant'Anna • Dany Flávio Tonelli • Teresa Cristina Monteiro Martins • Luiz Marcelo Antonialli

Tabela 2 Parques Tecnológicos em operação (até 2015) entrevistados na pesquisa

\begin{tabular}{|c|c|c|}
\hline Parque tecnológico & Cidade localizada & Estado \\
\hline BH-TEC & Belo Horizonte & Minas Gerais \\
\hline TecnoPARQ & Viçosa & Minas Gerais \\
\hline PCTI - Parque científico tecnológico de Itajubá & Itajubá & Minas Gerais \\
\hline Parque Tecnológico -RIO/UFRJ & Rio de Janeiro & Rio de Janeiro \\
\hline PÓLO BIO-RIO & Rio de Janeiro & Rio de Janeiro \\
\hline TECNÓPOLIS- Parque Tecnológico Região Serrana PET-TEC & Petrópolis & Rio de Janeiro \\
\hline CIATEC & Campinas & São Paulo \\
\hline PARQTEC - São Carlos Science Park & São Carlos & São Paulo \\
\hline Parque Tecnológico de São José dos Campos & São José dos Campos & São Paulo \\
\hline Parque Tecnológico UNIVAP & São José dos Campos & São Paulo \\
\hline Techno Park Campinas & Campinas & São Paulo \\
\hline Parque Tecnológico de Sorocaba & Sorocaba & São Paulo \\
\hline Parque Tecnológico Piracicaba "Engenheiro Agrônomo Emílio Bruno Germek" & Piracicaba & São Paulo \\
\hline Parque Tecnológico de Botucatu & Botucatu & São Paulo \\
\hline Fundação Parque Tecnológico da Paraíba - PaqTcPB & Campina Grande & Paraíba \\
\hline Parque Tecnológico da Bahia & Salvador & Bahia \\
\hline Porto Digital & Recife & Pernambuco \\
\hline SergipeTec & Aracaju & Sergipe \\
\hline Parque Tecnológico de Londrina Francisco Sciarra & Londrina & Paraná \\
\hline FUNDETEC & Cascavel & Paraná \\
\hline PTI Parque tecnológico Itaipu & Foz do Iguaçu & Paraná \\
\hline Parque Tecno-Científico da Unicentro -TECNICENTRO & Guarapuava & Paraná \\
\hline Programa Curitiba Tecnoparque & Curitiba & Paraná \\
\hline Parque de Software de Curitiba & Curitiba & Paraná \\
\hline $\begin{array}{l}\text { Associação de Desenvolvimento Tecnológico do Vale - VALETEC- Parque Tecnológico Vale dos } \\
\text { Sinos }\end{array}$ & Campo Bom & Rio Grande do Sul \\
\hline Parque Científico e Tecnológico da PUCRS (Tecnopuc) & Porto Alegre & Rio Grande do Sul \\
\hline Tecnosinos & São Leopoldo & Rio Grande do Sul \\
\hline UlbraTECH & Canoas & Rio Grande do Sul \\
\hline BIANCHINI BUSINESS PARK & Blumenau & Santa Catarina \\
\hline Sapiens Parque & Florianópolis & Santa Catarina \\
\hline ParqTec Alfa & Florianópolis & Santa Catarina \\
\hline INOVA PARQUE & JOINVILLE & Santa Catarina \\
\hline
\end{tabular}

Fonte Elaboração dos autores. 
Tabela 3 Comparação entre os grupos

\begin{tabular}{l|l|l}
\hline Grupo & Quanto ao reconhecimento da importância da GC & Quanto às ações que levam à GC \\
\hline Grupo 1 - Públicas & Menor importância & Mais ações em GC \\
\hline Grupo 2 - Privadas & Maior importância & Menos ações em GC \\
\hline
\end{tabular}

Fonte Elaboração dos autores.

Portanto, apesar dos atores públicos atribuírem menor importância às variáveis de colaboração, na prática, afirmam realizar mais ações colaborativas nos parques tecnológicos. O inverso acontece com os atores privados. Apesar de reconhecerem a importância das variáveis de colaboração afirmam que praticam menos ações nesse sentido. Denota-se que há significativa incoerência entre teoria e prática. Isso porque, torna-se curioso como os atores públicos podem afirmam que praticam ações colaborativas se não reconhecem a importância das mesmas. Nesse momento, também houve uma inquietude para se entender quais seriam as variáveis colaborativas mais ou menos reconhecidas e quais seriam mais ou menos praticadas pelos atores envolvidos.

Para tecer maiores considerações acerca desses grupos e identificar quais aspectos (variáveis) da colaboração são mais ou menos reconhecidas e praticadas pelos envolvidos, foi realizada a análise discriminante. Aplicando-se a análise discriminante das variáveis de importância em função dos clusters, constatou-se que 3 variáveis são as que melhor explicam a diferença entre os grupos. São elas: a gestão do conhecimento, a confiança e o investimento. A análise discriminante retornou um valor de correlação canônica de 0,825 e apresentou significância ao nível de 1\% (um por cento) pelo teste de Willks Lambda. Sendo assim, com base na correlação canônica ao quadrado, as variáveis destacadas explicam 68\% (sessenta e oito por cento) da diferença entre os grupos.

Por ordem de significância, as variáveis são ilustradas na tabela 4, ressaltando-se que entre parênteses encontra-se a porcentagem de entrevistados do grupo que deram a respectiva resposta.

Claramente, os atores privados entrevistados consideram a variável da gestão do conhecimento mais importante para a colaboração do que os atores públicos. Isso porque, $77 \%$ dos atores privados a consideram "muito importante", e somente 11,5\% dos atores públicos a consideram "muito importante". A maior parte dos atores públicos (69\%) dos atores públicos consideram a gestão do conhecimento "importante". Denota-se que a divergência entre o reconhecimento da importância da gestão do conhecimento na colaboração pode dar ensejo a controvérsias sociais entre os envolvidos, já que a integração contínua facilita, viabiliza e garante maior durabilidade à colaboração (WEBER; KHADEMIAN, 2008).

Por outro lado, Buuren (2009) pondera que apesar de reconhecer que a inclusão do conhecimento facilita a negociação entre as partes interessadas, esse elemento não é garantia de resultados positivos destas negociações. Isso porque, o conhecimento é uma das maiores fontes de fragmentação e discordância em redes de governança. Portanto, o autor entende que os componentes do conhecimento que estejam em conflito devem ser tratados para que as controvérsias sociais sejam cada vez menores. Esse tratamento deve ser realizado com a construção da confiança, estímulo à interação entre os atores e mobilização de profissionais competentes que orientem o processo de governança colaborativa (BUUREN, 2009).

Weber e Khademian (2008) também ressaltam que para se chegar no estágio do compartilhamento do conhecimento entre os vários envolvidos da rede colaborativa, o grau de confiança deve estar alto e consolidado. Nesse momento, verifica-se a total coerência desse raciocínio já que a variável da confiança apresentou significativa divergência entre os atores públicos e privados.

Dentre as instituições privadas, $89,6 \%$ entendem que a confiança na colaboração é um fator "muito importante", e somente $35,7 \%$ dos atores públicos entendem o mesmo. A maioria, ou seja, $58,6 \%$ das instituições públicas entendem que a confiança é um fator "importante" para a colaboração e ainda há aqueles que afirmam que a confiança não possui importância no processo colaborativo $(1,1 \%)$. Per- 
cebe-se que as mesmas instituições públicas que não reconhecem tanta importância na gestão do conhecimento são as mesmas que não apresentam um alto grau de reconhecimento da importância da confiança no processo colaborativo.

Por outro lado, mesmo que a confiança não exista desde o início ela pode ser construída durante o processo. Além disso, o nível de confiança nem sempre é o mesmo durante toda a colaboração (VAN OORTMERSSEN; VAN WOERKUM; AARTS, 2014). Portanto, mesmo que os atores não vislumbrem a grande importância que a confiança possui em processos colaborativos, é possível que essa percepção mude com a crescente interação entre os envolvidos na solução de problemas públicos que promovam ganhos para todos os envolvidos, como desenvolvimento local e geração de sistemas de inovação.

A variável do investimento para o desenvolvimento das pessoas e da infraestrutura do Parque, da mesma forma, apresentou significativa divergência de percepção entre atores públicos e privados. Apesar de 93,4\% das instituições privadas reconhecerem essa variável como "muito importante", nas instituição públicas 42,5\% entendem ser somente "importante". $\mathrm{O}$ o investimento é um fator muito importante para o início e a permanência da iniciativa colaborativa e assim como a gestão do conhecimento, depende de um ambiente de confiança. Os investimentos não são somente financeiros. Eles incluem bens tangíveis, tais como recursos financeiros, pessoas, tecnologia e suprimentos; e também os intangíveis, como conhecimento, status, identidade, ideologia, cultura e capacidades (TANG; TANG, 2014; PURDY, 2012). Para tanto, o governo local deve servir, nas palavras de Weber e Khademian (2008, p.341), "como um catalisador de parcerias", principalmente na presença de objetivos públicos na rede. Isso indica que governos municipais e estaduais podem atuar na atração de parcerias, seja por meio de investimentos, seja na construção da confiança para que outros atores também sejam somados às iniciativas de parque tecnológicos. Ressalta-se a ponderação de Almeida (2002, p. 15), sobre a necessidade de investimentos públicos para a pesquisa e a inovação, vez que "mesmo que a Universidade torne-se peça da engrenagem do mercado, o investimento público será sempre necessário, pois dificilmente as empresas investirão em pesquisas [...] incertas".

Por outro lado, é importante considerar que a equidade nos investimentos em ambientes colaborativos proporciona um maior equilíbrio inclusive quanto ao poder de influência de cada ator (Purdy, 2012). Portanto, se há uma primazia de investimentos para o setor público, este, por sua vez, poderá ditar a agenda de pesquisa e inovação ocasionando um desequilíbrio de poder na colaboração inviabilizando, inclusive, a permanência de parceiros (ANSELL; GASH, 2008).

Para comparar a classificação sobre o grau de importância atribuído às variáveis da GC e sua efetiva implementação nos parques, foi realizada também a análise discriminante com o objetivo de verificar quais seriam as variáveis que mais diferenciam os grupos 1 e 2 . Com nível de significância de $1 \%$ pelo teste de Willks Lambda e explicando 67\% da diferença entre os grupos, as variáveis que melhor diferenciam os grupos quanto ao estabelecimento concreto da GC nos parques são: multiplicidade de envolvidos, interdependência confiança, processo deliberativo e acordo formal, descritas na Tabela 4.

Denota-se que grande parte atores públicos (87\%) afirmam estar envolvidos com diversos tipos de instituições públicas e privadas, o que não acontece com as instituições privadas. Pouco mais da metade dos atores privados afirmam que se envolvem com diversas instituições e uma parte (11,5\%) afirma que não se envolve com outras instituições. Se o objetivo das iniciativas de parques tecnológicos é exatamente a união de esforços públicos e privados na promoção da inovação e do desenvolvimento local percebe-se que há pouco envolvimento do setor privado nessa interação. Esse resultado é preocupante, já que conforme Howlett (2014) é exatamente nessa variedade de diferentes atores interagindo uns com os outros ao longo do tempo, cada um com diferentes interesses e recursos, com limitações de conhecimento e informação, que se está a nova orientação de design de políticas públicas.

Outro resultado que chama a atenção é que enquanto 59,6\% das instituições públicas envolvidas nos Parques afirmam participar juntamente com outras instituições dos processos decisórios, 38,5\% dos atores privados afirmam que não participam, juntamente com outras instituições, dos processos 
Tabela 4 Principais dissensos de percepções sobre o processo colaborativo

\begin{tabular}{|c|c|c|}
\hline VARIÁVEL & GRUPO 1 - PÚBLICAS & GRUPO 2 - PRIVADAS \\
\hline $\begin{array}{l}\text { COMPARTILHAMENTO E TRANSFERÊNCIA DE CONHECI- } \\
\text { MENTO, SABERES E PRÁTICAS }\end{array}$ & $\begin{array}{l}\text { Pouco importante }(19,5 \%) \text { ou } \\
\text { importante }(69 \%)\end{array}$ & $\begin{array}{l}\text { Importante }(22,6 \%) \\
\text { Muito importante (77\%) }\end{array}$ \\
\hline CONFIANÇA ENTRE AS INSTITUICCÕES PARCEIRAS & $\begin{array}{l}\text { Sem importância }(1,1 \%) \text {, Pouco impor- } \\
\text { tante }(4,6 \%) \text { ou importante }(58,6 \%)\end{array}$ & $\begin{array}{l}\text { Importante }(10,4 \%) \\
\text { Muito importante }(89,6 \%)\end{array}$ \\
\hline $\begin{array}{l}\text { INVESTIMENTO para desenvolvimento das pessoas e da } \\
\text { infraestrutura do Parque }\end{array}$ & $\begin{array}{l}\text { Pouco importante }(5,7 \%) \text { ou } \\
\text { importante }(42,5 \%)\end{array}$ & $\begin{array}{l}\text { Importante }(6,6 \%) \\
\text { Muito importante }(93,4 \%)\end{array}$ \\
\hline $\begin{array}{l}\text { MULTIPLICIDADE- Estamos envolvidos com instituições } \\
\text { diversas }\end{array}$ & $\begin{array}{l}\text { Realiza parcialmente }(12,4 \%) \text { ou } \\
\text { Realiza (87\%) }\end{array}$ & $\begin{array}{l}\text { Não realiza }(11,5 \%) \\
\text { Realiza parcialmente }(54,8 \%)\end{array}$ \\
\hline Nossos resultados dependem da INTERDEPENDÊNCIA & $\begin{array}{l}\text { Realiza parcialmente }(22,5 \%) \text { ou } \\
\text { Realiza }(76,4 \%)\end{array}$ & $\begin{array}{l}\text { Não realiza }(15,4 \%) \\
\text { Realiza parcialmente }(45,2 \%)\end{array}$ \\
\hline Atuamos em um ambiente de CONFIANÇA & $\begin{array}{l}\text { Realiza parcialmente (36\%) ou } \\
\text { Realiza }(62,9 \%)\end{array}$ & $\begin{array}{l}\text { Não realiza (51\%) } \\
\text { Realiza parcialmente }(34,6 \%)\end{array}$ \\
\hline $\begin{array}{l}\text { PARTICIPAMOS juntamente com outras instituiç̧̃es dos } \\
\text { processos decisórios }\end{array}$ & $\begin{array}{l}\text { Realiza parcialmente }(34,8 \%) \text { ou } \\
\text { Realiza }(59,6 \%)\end{array}$ & $\begin{array}{l}\text { Não realiza }(38,5 \%) \\
\text { Realiza parcialmente (49\%) }\end{array}$ \\
\hline
\end{tabular}

Fonte Elaboração dos atores.

decisórios e $49 \%$ somente o fazem de maneira parcial. Sendo assim, somente $12,5 \%$ das empresas privadas (parceiras e instaladas no parque) afirmam participar com outras instituições dos processos decisórios dos parques do qual fazem parte. O resultado demonstra a não consolidação da deliberação e participação inclusiva nos parques tecnológicos em operação no país. Tal fator é preocupante, uma vez que o processo deliberativo inclusivo é tão importante que a literatura aponta seu papel influenciador sobre a construção da confiança, de forma que quanto maior for a participação, maior será a confiança entre os envolvidos (FREEMAN, 1997; MCDOUGALL et al, 2013; JOHNSTON et al, 2010). Observa-se essa ligação direta entre participação e confiança, pois da mesma forma que a participação se encontra comprometida, $51 \%$ das instituições privadas envolvidas no parques afirmam que não atuam em um ambiente de confiança, enquanto $62,9 \%$ das instituições públicas afirmam atuar em um ambiente de confiança. Portanto, a participação e a confiança são variáveis desafiantes para a colaboração nos parques tecnológicos em operação no Brasil, que podem comprometer sua durabilidade. A necessidade da melhoria no quesito de participação em um parque tecnológico no estado da Bahia também foi destacada no estudo de Marques, Costa, dos Santos e Orsatto (2017). Para os autores, se um dos principais objetivos de um parque tecnológico é promover a integração e a colaboração entre os diversos atores envolvidos, a participação possui relação direta com a transparência que favorece as relações de confiança e a troca de informações e conhecimento.

Com a variável da interdependência não é diferente. A prática de ações colaborativas de interdependência é diferente entre atores públicos e privados, já que somente $45,2 \%$ das empresas afirmam atuar de forma interdepende, enquanto $76,4 \%$ dos atores públicos afirmam o mesmo. A interdependência entre os atores significa que eles perceberão que sozinhos não poderão alcançar os objetivos da rede de colaboração, já que se for possível o alcance unilateral dos objetivos o processo colaborativo perderá sua razão de ser (ANSELL; GASH, 2008; EMERSON; NABATCHI; BALOGH, 2012; FREEDMAN, 1997; WEBER; LOVRICH; GAFFNEY, 2007; TANG; TANG, 2014). Por outro lado, se a maioria dos atores privados não atuam em um ambiente de confiança e menos da metade participam dos processos decisórios, dificilmente essa interdependência será percebida nas práticas colaborativas. Ademais, para que as regras e normas a serem seguidas pelos envolvidos sejam efetivamente cumpridas, elas precisam ser legitimadas por eles. Isso significa que cada envolvido teve a oportunidade de opinar e participar do processo de construção das regras (FREEMAN,1997; ULIBARRI, 
2015). Essa participação reforça o valor democrático que as partes dão às decisões e fortalece a interdependência (FREEMAN,1997). Portanto, se a participação resta comprometida, a interdependência segue o mesmo caminho.

\section{CONSIDERAÇÕES FINAIS}

O presente estudo identificou significativas diferenças de percepções quanto à importância dada por atores públicos e privados às variáveis da colaboração: gestão do conhecimento, confiança e investimento. Os atores privados reconhecem a maior importância dessas variáveis para o sucesso da colaboração nas iniciativas de parques tecnológicos no país. Por outro lado, apesar dos atores públicos atribuírem menor importância a tais variáveis, afirmam que praticam mais ações colaborativas do que os atores privados, referente às variáveis como: multiplicidade dos envolvidos, atuação em ambiente de confiança, a participação com outras instituições dos processos decisórios, e a existência da interdependência entre os envolvidos.

Portanto, a pesquisa demonstra que há significativas divergências entre as percepções e as práticas realizadas em parques tecnológicos por atores públicos e privados. $\mathrm{O}$ crescimento das iniciativas de parques tecnológicos dos país revelam uma predisposição à colaboração entre os envolvidos, no entanto o dissenso constatado entre atores públicos e privados é um desafio para a durabilidade desses empreendimentos. Se os atores públicos não reconhecem a grande importância da gestão do conhecimento, da atuação em um ambiente de confiança e da necessidade de investimentos para a colaboração, mesmo que haja objetivos comuns na criação de ambientes inovadores para a promoção do desenvolvimento local, a existência futura da colaboração e consequentemente dos próprios ambientes colaborativos de inovação pode ser comprometida pela falta de transparência e entendimento entre os envolvidos. Há uma relação direta entre teoria e prática, vez que a inter-relação entre as variáveis, apontada na literatura, foi confirmada no estudo. A falta de dos atores privados nas tomadas de decisões compromete a confiança entre os envolvidos. Se os atores privados encontram difi- culdades para atuarem em um ambiente de confiança, como fora evidenciado na pesquisa, não atuarão de forma interdependente e suas ações serão isoladas sem estreita relação com os objetivos públicos.

Este estudo contribui para a identificação das controvérsias existentes entre percepções e práticas colaborativas entre os atores públicos e privados envolvidos nos parques tecnológicos em operação no país. Os resultados apontam problemas quanto à ausência de ampla participação dos envolvidos nas tomadas de decisões, atuação em um ambiente de pouca confiança e interdependência e atribuição de pouca importância a ações de gestão do conhecimento e investimentos financeiros nos parques.

Pesquisas futuras podem ser empreendidas para se compreender as quais as razões que levam à menor percepção do grau de importância pelos atores públicos das variáveis da gestão do conhecimento, confiança e investimentos. Ademais, outras investigações podem identificar as causas da falta de participação dos atores privados nas tomadas de decisões.

Os autores agradecem o apoio da FAPEMIG para o desenvolvimento deste trabalho.

\section{REFERÊNCIAS}

ABDALLA, M. M.; CALVOSA, M. V. D.; BATISTA, L. G. Hélice tríplice no Brasil: um ensaio teórico acerca dos benefícios da entrada da universidade nas parcerias estatais. Revista Cadernos de Administração, São Paulo, 1 (3), 01-18, 2009

ABRAMOVSKY, Laura; SIMPSON, Helen. Geographic proximity and firm-university innovation linkages: evidence from Great Britain. Journal of Economic Geography, n.11, p. 949-977, 2011.

ALMEIDA, M. L. P. Universidade e iniciativa privada: a UNICAMP diante dos desafios da globalização. Campinas: Alínea, 2002. 
ANPROTEC, Associação Nacional de Entidades Promotora de Empreendimentos Inovadores. Estudo de Projetos de Alta Complexidade: Indicadores de Parques Tecnológicos - Versão final. 2014. Disponível em: $<$ http://www.anprotec.org.br/Relata/ PNI_FINAL_web.pdf $>$ Acesso em 28 nov., 2017a.

ANPROTEC, Associação Nacional de Entidades Promotora de Empreendimentos Inovadores. Propostas de Políticas Públicas para Parques Tecnológicos e Incubadora de Empresas. 2015. Disponível em: < http://ppi.certi.org.br/4-PropostasPoliticasPublicasParquesIncubadoras.pdf > Acesso em 28 nov. 2017b.

ANSELL, Chris; GASH, Alison. Collaborative governance in theory and practice. Journal of Public Administration Research and Theory, v. 18, n. 4, p. 543-571, Oct 2008.

APPLEYARD, M. M.; CHESBROUGH, H. W. The Dynamics of Open Strategy: From Adoption to Reversion. Long Range Planning, 50, 310-321, 2017.

CHILD, J.; FAULKNER, D.; TALLMAN, S. Cooperative strategy. New York: Oxford University Press, 2005.

CHOI, Tae Hyon; ROBERTSON, Peter J. Caucuses in Collaborative Governance: Modeling the Effects of Structure, Power, and Problem Complexity. International Public Management Journal, v.17, n.2, p. 224-254, 2014.

DYER, J. H.; SINGH, H. The relational view: cooperative Strategy and sources of interorganizational competitive Advantage. Academy of Management Review, 23 (4), 660-679. 1998.

EMERSON, Kirk; NABATCHI, Tina; BALOGH, Stephen. An Integrative Framework for Collaborative Governance. Journal of Public Administration Research and Theory, v. 22, n. 1, p. 1-29, Jan 2012.
ETZKOWITZ, H.; LEYDESDORFF, L. The dynamics of innovation: from National Systems and "Mode 2" to a Triple Helix of university-industry-government relations. Research Policy, Amsterdam, 29 (2), 109123, 2000.

FOSTER-FISHMAN, Pennie G.; BERKOWITZ, Shelby L.; LOUNSBURY, David W. Building Collaborative Capacity in Community. American Journal of Community Psychology, Vol. 29, N. 2,241-261, 2001.

FREEMAN, Jody. Collaborative governance in the administrative state. Ucla Law Review, v. 45, n. 1, p. 1-98, Oct 1997.

GUILLAIN, R., HURIOT, J.M. The local dimension of information spillovers: a critical review of empirical evidence in the case of innovation. Canadian Journal of Regional Science, 24 (2), 313-338, 2001.

HAIR, Joseph F.; ANDERSON, Rolph L.; TATHAN, Ronald L.; BLACK, William C. Análise multivariada de dados. Porto Alegre: Bookman, 2005.

HANSEN, Peter Bent; BECKER, Grace Vieira; NEFF, Henrique Bagattini; MELLO, Natália Coll. Contribuição do parque tecnológico para a competitividade das empresas instaladas: análise do caso do Tecnopuc - RS. Revista Gestão Organizacional, vol. 5, n. 2, jul./dez, 2012.

HERVÁS-OLIVER, Jose Luis; ALBORS-GARRIGOS, Jose. The role of firm's internal and relational capabilities in clusters: when distance and embeddedness are not enough to explain innovation. Journal of Economic Geography, Vol. 9, n. 2, p. 263-283, 2009.

HOBBS, K. G.; LINK, A. N.; SCOTT, J. T. Science and technology parks: an annotated and analytical literature review. Journal of Technology Transfer, vol. 42, p 957-976, 2017.

HOWLETT, M. From the 'old' to the 'new' policy design: design thinking beyond markets and collaborative governance. Policy Science, 47, p. 87-207, 2014. 
IZUSHI, Hiro. Impact of the length of relationships upon the use of research institutes by SMEs. Research Policy, n. 32, p.771-788, 2003.

JOHNSTON, Erik W.; HICKS, Darrin; NAN, Ning; AUER, Jennifer C. Managing the Inclusion Process in Collaborative Governance. Journal of Public Administration Research and Theory.21 (4), p. 699721, august, 2010.

KALLIS, Giorgos; KIPARSKY, Michael; NORGAARD, Richard. Collaborative governance and adaptive management: Lessons from California's CALFED Water Program. Environmental Science \& Policy, v. 12, n. 6, p. 631-643, Oct 2009.

LACERDA, Norma; FERNANDES, Ana Cristina. Parques tecnológicos: entre inovação e renda imobiliária no contexto da cidade do Recife. Cadernos Metrópole, v. 17, n. 34, pp. 329-354, nov 2015.

LAIMER, Claudionor Guedes. Determinants of interorganizational relationships in science and technology parks: theoretical and empirical evidence. Gestão \& Regionalidade, vol. 31, n.91, jan-abr, 2015.

MAH, Daphne Ngar-Yin; HILLS, Petter. Collaborative governance for technological innovation: a comparative case study of wind energy in Xinjiang, Shanghai, and Guangdong. Environment and Planning C: Government and Policy, v.32, n.3, p.509-529, 2014.

MARQUES, M.A.J.; COSTA, V.A.; DOS SANTOS, R.C.P.; ORSATTO, S.G. Análise de percepção de atores de Ciência, Tecnologia e Inovação em sistemas regionais

e ambientes de inovação. Revista de Gestão e Tecnologia, 7 (3), 86-94, 2017.

MCDOUGALL, Cynthia L.; LEEUWIS, Cees; BHATTARAI, Tara; MAHARJAN, Manik R.; JIGGINS, Janice; Engaging women and the poor: adaptive collaborative governance of community forests in Nepal. Agriculture and Human Values, v. 30, n. 4, p 569- 585, 2013.
MOELIODIHARDJO, B. Y; SOEMARDI, B.W.; BRODJONEGORO, S.S. \& HATAKENAKA, S. University, industry, and government partnership: its present and future challenges in Indonesia. Procedia Social and Behavioral Sciences, 52, 307-316, 2012.

MOREIRA;,S.T.B.; TONELLI, D.F.; MESQUISTA, I.; SILVA, S.R.; V, M.O.S. Inovação no Setor Público: explorando possibilidades a partir de uma Proposta de Diagrama Analítico. In: VI Encontro de Administração Pública e Governança (EnAPG), 2014, Belo Horizonte. Anais do VI Encontro de Administração Pública e Governança (EnAPG), 2014.

NEWMAN, Janet; BARNES, Marian; SULLIVAN, Helen; KNOPS, Andrew. Public Participation and Collaborative Governance. Journal of Social Policy, V. 33 (2), 203 - 223, April 2004.

OLIVEIRA, S. C. Os caminhos para a inovação tecnológica e o papel da universidade nesse processo. In: Gazzola, A. L. A.; Almeida, S. G. (Org.). Universidade: cooperação internacional e diversidade. Belo Horizonte: Editora da UFMG, 205-211, 2006.

PESSOA Leonel Cesarino; BRITO, Silva Cirani Claudia ; MUNIZ, Silva Marcello; SOUZA, Rangel Armênio. Parques tecnológicos brasileiros: uma análise comparativa de modelos de gestão. Revista de Administração e Inovação, São Paulo, v. 9, n. 2, p.250-270, abr/jun. 2012.

PEREIRA, Alexandre. Guia prático de utilização do SPSS. Análise de dados para ciências sociais e psicologia, 2004.

PURDY, Jill M. Framework for Assessing Power in Collaborative Governance Processes. Public Administration Review, 72 (3), p. 409-417, Mayjun. 2012.

ROBERTSON, Peter J.; CHOI, Taehyon Deliberation, Consensus, and Stakeholder Satisfaction. Public Management Review, 14 (1), p. 83-103, 2012. 
RODRIGUES, C.; MELO, A. I. The triple helix model as inspiration for local development policies: an experience-based perspective. International Journal of Urban and Regional Research, 37 (5), 1675-1687, 2013.

SANT'ANNA, Lindsay. T.; TONELLI, Dany F.; ABBUD, Elenice. B. Collaborative Governance: a Maturity Level Proposal based on a Scoping Study. XL Encontro da ANPAD. Costa do Sauípe, setembro, 2016. Anais...Costa do Sauípe, 2016. p.1-16.

SCHMIDT, Serje; BALESTRIN, Alsones. Projetos colaborativos de $\mathrm{P} \& \mathrm{D}$ em ambientes de incubadoras e parques científico-tecnológicos: teorizações do campo de estudo. Revista de Administração e Inovação, v. 11, n.2, p. 111-131, abr./jun. 2014.

SIDDIKI, S.N.; CARBONI, J.L.; KOSKI, C.; SADIQ, A-A. How Policy Rules Shape the Structure and Performance of Collaborative Governance Arrangements. Public Administration Review, 75 (4), p. 536-547, jul-aug, 2015.

SILVA, Fabio Q. B.; SUASSUNA, Marcos; MACIEL, Sheyla de Moraes. Um modelo de desenvolvimento local baseado em inovação e o papel dos parques tecnológicos na sua implantação. Revista da Micro e Pequena Empresa, v.3, n.1, p.25-37, 2009.

STEINER, J. E.; CASSIN, M. B.; ROBAZZI, A. C. Parques tecnológicos: ambientes de inovação. São Paulo: Instituto de Estudos Avançados de São Paulo. 2008.

TANG, C-P.; TANG, S-Y. Managing Incentive Dynamics for Collaborative Governance in Land and Ecological Conservation. Public Administration Review, 74 (2), p. 220-231, 2014.

TENCATI, A.; ZSOLNAI, L. The Collaborative Enterprise. Journal of Business Ethics, v. 85, p. 367-376, 2009.
TONELLI, Danny Flávio; MARQUESINI, Matheus Almeida; ZAMBALDE, André Luiz. Implantação de Parques Tecnológicos como Política Pública: Uma Revisão Sistemática sobre seus Limites e Potencialidades. Revista Gestão \& Tecnologia, v. 15, n. 2, p. 113-134 mai./ago. 2015.

TONELLI, D. F.; SANT'ANNA, L.; ABBUD, E. B; SOUZA, S. A. S. Antecedents, process, and equity outcomes: A study about collaborative governance. Cogent Business \& Management, 5, 1-17, 2018.

VÁSQUEZ-URRIAGO, Ángela Rocío; BARGEGIL, Andrés; RICO, Aurelia Modrego. Science and Technology Parks and cooperation for innovation: Empirical evidence from Spain. Research Policy, vol. 45, 137-147, 2016.

VEDOVEllo, Conceição Aparecida; JUDICE, Valéria; MACULAN, Anne-Marie. Revisão crítica às abordagens a parques tecnológicos: alternativas interpretativas às experiências brasileiras recentes. RAI - Revista de Administração e Inovação, v. 3, n. 2, p. 103-118, 2006.

WEBER, E.P.; LOVRICH, N.P.; GAFFNEY, M.J.; Assessing Collaborative Capacity in a Multidimensional World. Administration \& Society, 39(2), 194-220, April 2007.

WEBER, Edward P.; KHADEMIAN, Anne M.; Wicked Problems, Knowledge Challenges, and Collaborative Capacity Builders in Network Settings. Public Administration Review, 334-349, march/ april 2008.

ZEN, A. C. A articulação e o desenvolvimento dos parques tecnológicos: O caso do Programa Porto Alegre Tecnópole - Brasil. In: XI Seminário Latino Americano de Gestión Tecnológica. Anais... Porto Alegre. Outubro de 2005.

ZOUAIN, Desiree Morais; PLONSKI, Guilherme Ary. Parques tecnológicos - planejamento e gestão. Brasília: ANPROTEC, SEBRAE. 2006. 